\title{
SMA observations of young disks: separating envelope, disk, and stellar masses in class I YSOs
}

\author{
D. Lommen ${ }^{1}$, J. K. Jørgensen ${ }^{2,3}$, E. F. van Dishoeck ${ }^{1}$, and A. Crapsi ${ }^{1,4}$ \\ 1 Leiden Observatory, Leiden University, PO Box 9513, 2300 RA Leiden, The Netherlands \\ e-mail: dave@strw.leidenuniv.nl \\ 2 Harvard-Smithsonian Center for Astrophysics, 60 Garden Street, Cambridge, MA 02138, USA \\ 3 Argelander-Institut für Astronomie, University of Bonn, Auf dem Hügel 71, 53121 Bonn, Germany \\ 4 Observatorio Astronómico Nacional (IGN), Alfonso XII, 3, 28014 Madrid, Spain
}

Received 26 March 2007 / Accepted 14 January 2008

\section{ABSTRACT}

\begin{abstract}
Context. Young stars are born with envelopes, which in the early stages obscure the central (proto)star and circumstellar disk. In the Class I stage, the disks are still young, but the envelopes are largely dispersed. This makes the Class I sources ideal targets for studies of the early stages of disks.

Aims. We aim to determine the masses of the envelopes, disks, and central stars of young stellar objects (YSOs) in the Class I stage. Methods. We observed the embedded Class I objects IRS 63 and Elias 29 in the $\rho$ Ophiuchi star-forming region with the Submillimeter Array (SMA) at $1.1 \mathrm{~mm}$.

Results. IRS 63 and Elias 29 are both clearly detected in the continuum, with peak fluxes of 459 and $47 \mathrm{mJy} / \mathrm{beam}$, respectively. The continuum emission toward Elias 29 is clearly resolved, whereas IRS 63 is consistent with a point source down to a scale of $3^{\prime \prime}$ (400 AU). The SMA data are combined with single-dish data, and both disk masses of 0.055 and $\leq 0.007 M_{\odot}$ and envelope masses of 0.058 and $\leq 0.058 M_{\odot}$ are empirically determined for IRS 63 and Elias 29, respectively. The disk+envelope systems are modelled with the axisymmetric radiative-transfer code RADMC, yielding disk and envelope masses that differ from the empirical results by factors of a few. $\mathrm{HCO}^{+} J=3-2$ is detected toward both sources, $\mathrm{HCN} J=3-2$ is not. The $\mathrm{HCO}^{+}$position-velocity diagrams are indicative of Keplerian rotation and allow an estimate of the mass of the central stars. For a fiducial inclination of $30^{\circ}$, we find stellar masses of $0.37 \pm 0.13$ for IRS 63 and $2.5 \pm 0.6 M_{\odot}$ for Elias 29 .

Conclusions. The sensitivity and spatial resolution of the SMA at $1.1 \mathrm{~mm}$ allow a good separation of the disks around Class I YSOs from their circumstellar envelopes and environments, and the spectral resolution makes it possible to resolve their dynamical structure and estimate the masses of the central stars. The ratios of the envelope and disk masses $M_{\text {env }} / M_{\text {disk }}$ are found to be 0.2 for IRS 63 and 6 for Elias 29. This is lower than the values for Class 0 sources, which have $M_{\text {env }} / M_{\text {disk }} \geq 10$, suggesting that this ratio is a tracer of the evolutionary stage of a YSO.
\end{abstract}

Key words. circumstellar matter - stars: formation - stars: individual: IRS 63 - stars: individual: Elias 29

\section{Introduction}

Low- and intermediate-mass stars are formed from the gravitational collapse of molecular cloud cores. In the earliest stages, the newly-formed protostar remains embedded in the remnants of this core, a cold envelope of dust and gas, which is gradually accreted by the young star (e.g., Shu 1977). Due to the angular momentum initially present in the core, most of the envelope material does not fall directly onto the central protostar but is piled-up in a circumstellar disk (e.g., Terebey et al. 1984). Understanding this interplay between star, disk, and envelope is crucial in order to be able to relate the initial conditions of star formation such as the mass of the protostellar core to the endproduct - namely the properties of the young star, and the mass and thus potential of the disk for forming planets. Some of the key questions include: Where does most of the mass reside at a given time? Will all the mass that is seen in prestellar cores or in the envelopes around deeply embedded sources end up in the star, or will a large fraction be dispersed from the system? How do the masses of the circumstellar envelope, the disk, and that of the central star evolve over time, and how long does it take for the circumstellar matter to be accreted onto the star?
Young stellar objects (YSOs) are usually classified according to their slopes in the infrared (IR) wavelength regime. Originally, the LW classification (Lada \& Wilking 1984; Lada 1987) ran from the embedded Class I, via the optically visible Class II or classical T Tauri stars, to the Class III spectral energy distributions of post-T Tauri stars. Later, the Class 0 stage was added to this classification (see, e.g., André et al. 1993), where the Class 0 sources are distinguished from the Class I sources through their high relative luminosity at submillimetre (submm) wavelengths. This classification roughly reflects the evolutionary stage of the YSOs under consideration. The most deeply embedded Class 0 sources are thought to evolve through the Class I stage while dissipating their circumstellar envelopes. Eventually they become optically visible as pre-main sequence T Tauri stars with circumstellar disks.

The Class 0 and Class II YSOs have been studied quite extensively with high-resolution (sub)millimetre interferometers (e.g., Jørgensen et al. 2007; Andrews \& Williams 2007, and references therein). Studies of deeply embedded Class 0 YSOs have shown that circumstellar disks are formed early (Harvey et al. 2003; Jørgensen et al. 2004), but in these systems, it is difficult 
to separate the emission from the disk from that of the envelope. The Class I sources, in which a large part of the original circumstellar envelopes has been dissipated, are ideal to study young disks in YSOs. Interferometric studies of Class I objects have so far been largely limited to studies at around $3 \mathrm{~mm}$ (e.g., Ohashi et al. 1997; Hogerheijde et al. 1997, 1998; Looney et al. 2000). The Submillimeter Array (SMA) allows observations around $1 \mathrm{~mm}$, where the thermal dust continuum emission is an order of magnitude stronger than at $3 \mathrm{~mm}$. Also at these shorter wavelengths it is possible to detect the higher rotational transitions of the molecules that trace the dense gas in the disks and inner envelopes of the young systems, rather than lower-density extended envelope emission.

We here present SMA observations of two Class I objects that appear to be in an evolutionary stage where the kinematics of the circumstellar material are no longer dominated by infall. IRS 63 (WLY 2-63, GWAYL 4) and Elias 29 (Elia 2-29, WLY 1-7) are located in the $\rho$ Ophiuchi cloud, taken to be at a distance $D=125 \pm 25$ pc (de Geus et al. 1989). Spitzer photometry from the "Cores to Disks" Legacy program (Evans et al. 2003) was used to determine the source's infrared colours and their bolometric luminosities and temperatures. IRS 63 and Elias 29 have similar bolometric temperatures, $T_{\text {bol }}=351 \mathrm{~K}$ and $391 \mathrm{~K}$, respectively, which places them in the LW Class I regime. The bolometric luminosities were calculated to be $L_{\text {bol }}=0.79 L_{\odot}$ for IRS 63 and $13.6 L_{\odot}$ for Elias 29. According to their infrared slopes of $\alpha_{2-24 \mu \mathrm{m}}=0.15$ and 0.42 , IRS 63 is the slightly more evolved, falling into the "flat-spectrum" class that separates the Class I from the Class II sources (Greene et al. 1994). The values for $\alpha_{\mathrm{IR}}$ quoted here are from un-dereddened observations. Correction for the extinction toward the $\rho$ Ophiuchi star-forming region (e.g., Flaherty et al. 2007) would result in lower values for $\alpha_{\mathrm{IR}}$; in other words, the sources may be slightly more evolved than the raw values of $\alpha_{\mathrm{IR}}$ suggest. In terms of environment, the two sources are quite each other's opposites. The SCUBA $850 \mu \mathrm{m}$ map (Johnstone et al. 2004) from the COMPLETE survey (Ridge et al. 2006) shows that IRS 63 is an isolated compact source. Elias 29, on the other hand, is located in a dense ridge of molecular material, which contains several more YSOs. It is likely that these YSOs were formed from condensations in this molecular ridge.

We present the masses of all main components of Class I YSOs, i.e., the central star, the disk, and the envelope, for the first time. IRS 63 and Elias 29 are the first two sources in a larger survey of Class I objects studied with the SMA at $1.1 \mathrm{~mm}$. These observations complement the survey of Class 0 sources in the PROSAC programme (Jørgensen et al. 2007) and will allow us to trace the similarities and differences of these evolving protostars. The results of the complete campaign will be presented in a future paper. In Sect. 2, the observations are presented, and the results and implications are discussed in Sects. 3 and 4. We summarise the main conclusions in Sect. 5.

\section{Observations}

IRS 63 and Elias 29 were observed with the SMA ${ }^{1}$ (Ho et al. 2004) on 15 and 17 May 2006, respectively. Weather conditions were good on 15 May, with zenith optical depths at $225 \mathrm{GHz} \tau_{225}=0.04-0.06$ (as measured by a tipping radiometer

\footnotetext{
1 The Submillimeter Array is a joint project between the Smithsonian Astrophysical Observatory and the Academia Sinica Institute of Astronomy and Astrophysics and is funded by the Smithsonian Institution and the Academia Sinica.
}

Table 1. Results of SMA observations at $1.1 \mathrm{~mm}$.

\begin{tabular}{|c|c|c|c|}
\hline & IRS 63 & $\overline{E l i a s} 29$ & Ridge \\
\hline$F_{v}(\mathrm{P})^{a}\left[\mathrm{mJy} \mathrm{bm}^{-1}\right]$ & 459 & 47 & 31 \\
\hline $\mathrm{RA}^{a}[\mathrm{~J} 2000]$ & $16: 31: 35.65$ & $16: 27: 09.42$ & $16: 27: 09.48$ \\
\hline $\operatorname{Dec}^{a}[\mathrm{~J} 2000]$ & $-24: 01: 29.56$ & $-24: 37: 18.91$ & $-24: 37: 22.48$ \\
\hline $\mathrm{rms}^{b}\left[\mathrm{mJy} \mathrm{bm}^{-1}\right]$ & 6 & 5 & 5 \\
\hline \multicolumn{4}{|c|}{ Circular Gaussian fits to all data } \\
\hline$F_{v}(\mathrm{G})^{c}[\mathrm{mJy}]$ & 474 & 72 & 28 \\
\hline$F W H M^{c}[\operatorname{arcsec}]$ & $0.55 \pm 0.08$ & $2.2 \pm 0.5$ & - \\
\hline \multicolumn{4}{|c|}{ Circular Gaussian fits to data $\geq 16 \mathrm{k} \lambda$ (scales $<13$ arcsec) } \\
\hline$F_{v}(\mathrm{G})^{c}[\mathrm{mJy}]$ & 480 & 57 & 27 \\
\hline$F W H M^{c}[\operatorname{arcsec}]$ & $0.62 \pm 0.08$ & $1.7 \pm 0.7$ & - \\
\hline \multicolumn{4}{|l|}{ Line data } \\
\hline $\mathrm{HCO}^{+d}\left[\mathrm{~K} \mathrm{~km} \mathrm{~s}^{-1}\right]$ & $3.1 \pm 0.3$ & $23.7 \pm 2.5$ & $23.5 \pm 2.5$ \\
\hline $\mathrm{HCN}^{d}\left[\mathrm{~K} \mathrm{~km} \mathrm{~s}^{-1}\right]$ & $<1.8$ & $<3.8$ & $<3.8$ \\
\hline
\end{tabular}

${ }^{a}$ Point source fit in the $(u, v)$ plane.

${ }^{b}$ Calculated from the cleaned image.

${ }^{c}$ Circular Gaussian fit in the $(u, v)$ plane.

${ }^{d}$ Integrated intensities are from a $4 \times 4$ " square around the continuum emission. The synthesised beam is $\sim 4 \times 2.5^{\prime \prime}$. HCN was not detected; quoted values are $3 \sigma$ upper limits.

operated by the Caltech Submillimeter Observatory). Conditions were slightly worse on 17 May, with $\tau_{225}$ starting at 0.16 , falling to 0.1 during the first half of the night. Physical baselines ranged from 11.6 to $62.0 \mathrm{~m}$, and the resulting projected baselines ranged from 5.9 to $63.8 \mathrm{k} \lambda$, yielding a resolution of about $4.0 \times 2.3^{\prime \prime}$ (natural weighting). The correlator was configured to observe the lines of $\mathrm{HCO}^{+} J=3-2$ and $\mathrm{HCN} J=3-2$ together with the continuum emission at 268 and $278 \mathrm{GHz}$. The line data were taken with 512 channels of $0.2 \mathrm{MHz}$ width each, resulting in a velocity resolution of $0.23 \mathrm{~km} \mathrm{~s}^{-1}$.

Calibration was done using the MIR package (Qi 2005), and imaging was done using the MIRIAD package (Sault et al. 1995). The quasars QSO B1622-297 and QSO B1514-241 served as gain calibrators, and the absolute fluxes were calibrated on Uranus. The absolute flux calibration is estimated to have an uncertainty of $\sim 20 \%$. The passbands were calibrated on Callisto and the quasar QSO B1253-055 (3C 279).

\section{Results}

The basic results of the observations are shown in Fig. 1 and summarised in Table 1.

\subsection{Continuum data}

Both sources are clearly detected in the continuum at $1.1 \mathrm{~mm}$. Point-source and Gaussian fits were done in the $(u, v)$ plane to determine the peak and integrated fluxes, respectively. A continuum peak flux of $459 \mathrm{mJy} \mathrm{bm}^{-1}$ and an integrated flux of 474 mJy were found for IRS 63, at a position which agrees with the 2 MASS $\mathrm{K}_{\mathrm{S}}$-band position within 0'. 1 . IRS 63 was also observed with the SMA by Andrews \& Williams (2007) at higher resolution at $1.3 \mathrm{~mm}$. Their interferometer flux for IRS 63, to which they refer as L1709 $\mathrm{B}^{2}$, is consistent with the value here and a mm slope $\alpha_{\mathrm{mm}}=2.0 \pm 1.0$. The data of Elias 29 are best

2 IRS 63 and L1709 B are often referred to as the same source (e.g., André \& Montmerle 1994). However, L1709 B would appear to lie about 4 arcmin north of IRS 63 (e.g., Benson \& Myers 1989). The source that Andrews \& Williams (2007) refer to as L1709 B, is in fact IRS 63. 


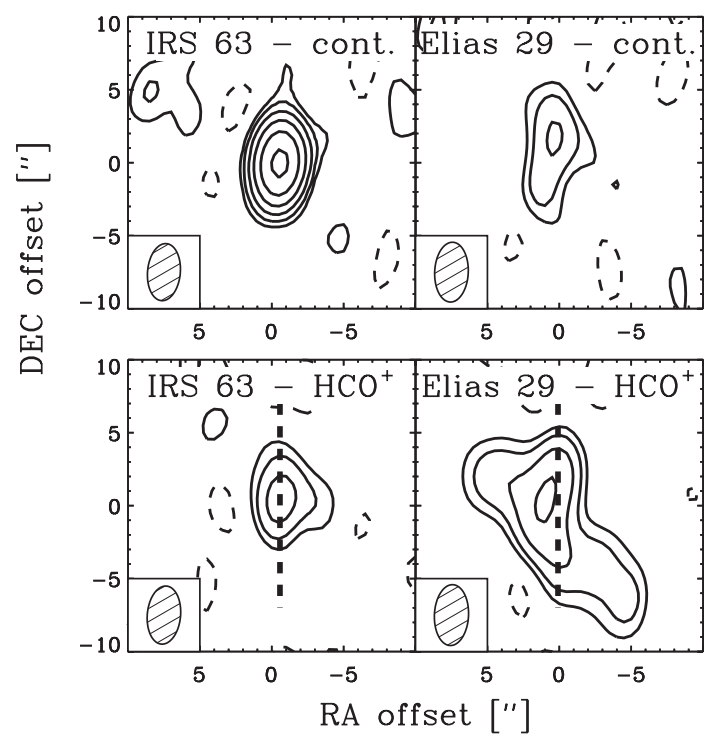

Fig. 1. SMA images of the $1.1 \mathrm{~mm}$ continuum (top panels) and integrated $\mathrm{HCO}^{+} 3-2$ (bottom panels) emission. Contours are drawn at 2, $4,8,16,32$, and 64 times the respective rms noise levels, with the rms being about $6 \mathrm{mJy} \mathrm{bm}^{-1}$ for the continuum and about $65 \mathrm{mJy} \mathrm{bm}^{-1}$ for the line data. Negative contours are dashed. The positional offsets are with respect to the coordinates of the observational phase centre. The dashed lines indicate how the position-velocity diagrams (Fig. 7) are directed.

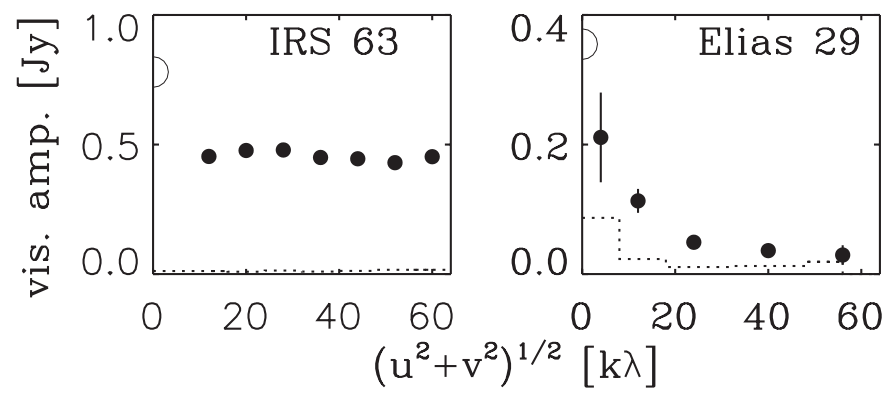

Fig. 2. Continuum amplitude as a function of projected baseline for IRS 63 (left panel) and Elias 29 (right panel). The data points give the amplitude per bin, where the data are binned in annuli according to $(u, v)$ distance. The error bars show the statistical $1 \sigma$ errors, most often smaller than the data points, and the dotted lines give the expected amplitude for zero signal, i.e., the anticipated amplitude in the absence of source emission. The half-open circles at zero $(u, v)$ distance give the zero-spacing $1.1 \mathrm{~mm}$ flux, interpolated between $850 \mu \mathrm{m}$ and $1.25 \mathrm{~mm}$ single-dish fluxes (André \& Montmerle 1994; Johnstone et al. 2004; Ridge et al. 2006, see text).

fitted by two sources. The brightest of the two agrees with the 2MASS $K_{\mathrm{S}}$-band position of Elias 29 within 0 '.2, whereas the other source is offset by $3^{\prime \prime}$. The second peak is attributed to an enhancement in the ridge, from which Elias 29 likely formed, and is designated by "Ridge" in Table 1. Elias 29 and the ridge component yield continuum peak fluxes of 47 and $31 \mathrm{mJy} \mathrm{bm}^{-1}$, and integrated fluxes of 72 and $28 \mathrm{mJy}$, respectively.

Plots of the visibility amplitudes as functions of projected baseline for IRS 63 and Elias 29 are shown in Fig. 2. Elias 29 shows a steeply rising flux toward the shortest baselines, indicating that an envelope is present around this source. The emission toward IRS 63 does not change appreciably with baseline length, indicating that this source is unresolved up to baselines of $60 \mathrm{k} \lambda$, or down to physical scales of about $400 \mathrm{AU}$.

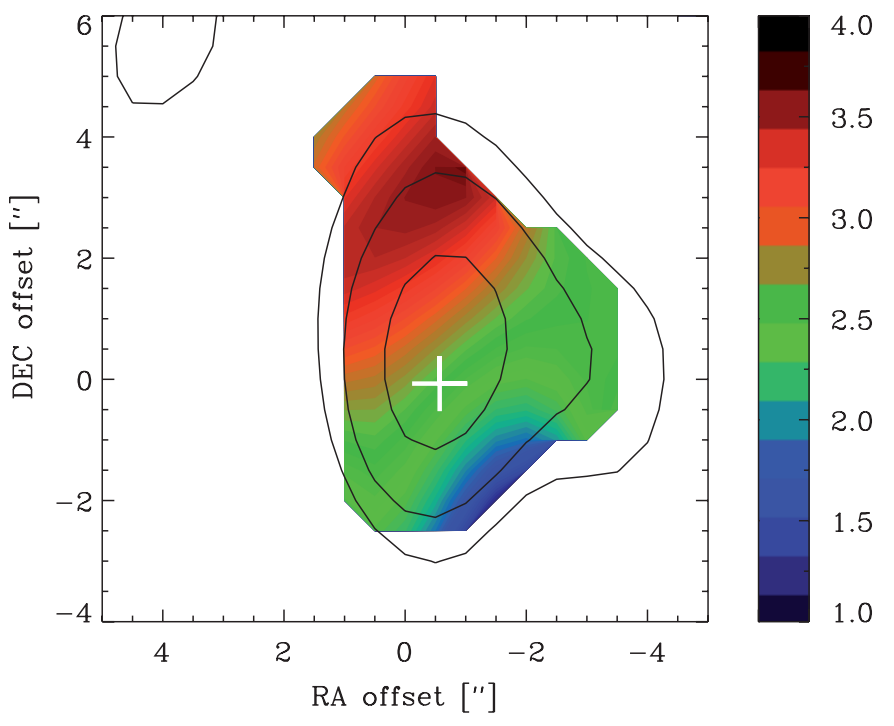

Fig. 3. First-moment map of IRS 63. Contours are drawn at 2, 4, and 8 times the RMS of $67 \mathrm{mJy} \mathrm{bm}^{-1}$. The positional offsets are with respect to the coordinates of the observational phase centre, and the cross indicates the continuum position of IRS 63 from a point-source fit in the $(u, v)$ plane, see Table 1. A colour version of this figure can be obtained from the electronic version of the paper.

Figure 2 also shows the estimated single-dish flux at $1.1 \mathrm{~mm}$. For this, the "mapped" fluxes from André \& Montmerle (1994) at $1.25 \mathrm{~mm}$ were combined with newly determined $850 \mu \mathrm{m}$ fluxes (SCUBA maps from the COMPLETE survey, Johnstone et al. 2004; Ridge et al. 2006). The SEDs are assumed to have a $F_{v} \propto v^{\alpha}$ dependence in this wavelength range, and are interpolated to the $1.1 \mathrm{~mm}$ wavelength at which the SMA observations were conducted. Fluxes of 355 and $780 \mathrm{mJy}$ are found for Elias 29 and IRS 63, respectively, with an estimated uncertainty of $25 \%$. These fluxes are significantly larger than the interferometer fluxes and indicate that extended emission is present around the compact components picked up with the SMA. This extended emission may be due to a circumstellar envelope, or to surrounding or intervening interstellar clouds, since the "mapped" regions of André \& Montmerle (1994) are rather large, 40" for Elias 29 and 60" for IRS 63.

\subsection{Line data}

$\mathrm{HCO}^{+} J=3-2$ is detected toward both sources, whereas $\mathrm{HCN}$ $J=3-2$ is not. Elias 29 appears to be about ten times as strong in $\mathrm{HCO}^{+} J=3-2$ integrated emission as IRS 63 (see Table 1). The emission toward IRS 63 is compact, coincident with the continuum peak, and shows a velocity gradient in the north-south direction, see Fig. 3. The integrated emission in the direction of Elias 29 is more extended and can be fitted with two elliptical Gaussians, as is shown in the left panel of Fig. 4. The centre position of the smaller Gaussian coincides with the continuum peak, as well as with the infrared position, and is attributed to Elias 29 itself. The second Gaussian has a very elongated shape, offset from the infrared source, and is attributed to a density enhancement in the nearby ridge. The right panel of Fig. 4 shows the $\mathrm{HCO}^{+} J=3-2$ spectra toward the continuum positions of Elias 29 and IRS 63, binned to $0.9 \mathrm{~km} \mathrm{~s}^{-1}$. The $\mathrm{HCO}^{+} J=3-2$ emission toward IRS 63 appears to be centred at $V_{\mathrm{LSR}}=3.3 \mathrm{~km} \mathrm{~s}^{-1}$. 


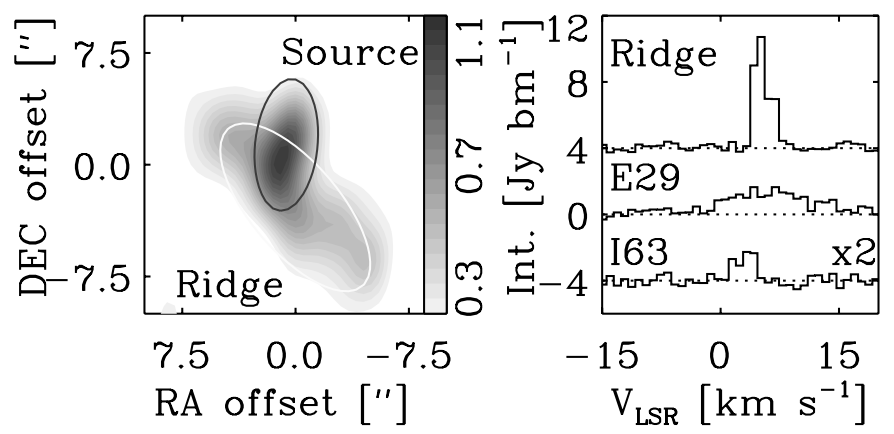

Fig. 4. Left panel: $\mathrm{HCO}^{+} J=3-2$ integrated emission toward Elias 29 . The grayscale is linear from 0.2 to $1.2 \mathrm{Jy} \mathrm{bm}^{-1}$. The emission is fitted with two Gaussians, the one in the north-south direction tracing the emission due to Elias 29, and the other, larger one tracing emission from a density enhancement in the ridge. Right panel: spectral lines, integrated over $2 \times 2^{\prime \prime}$ regions, centred on the continuum positions of Elias 29, of the ridge (shifted by $+4 \mathrm{Jy} \mathrm{bm}^{-1}$ ), and of IRS 63 (shifted by $-4 \mathrm{Jy} \mathrm{bm}^{-1}$ ), and binned to $0.9 \mathrm{~km} \mathrm{~s}^{-1}$. The $\mathrm{HCO}^{+} J=3-2$ emission toward IRS 63 appears to be centred at $V_{\mathrm{LSR}}=3.3 \mathrm{~km} \mathrm{~s}^{-1}$.

\section{Discussion and interpretations}

\subsection{Envelope and disk masses}

The basic data as presented in the previous section are used to determine the masses of the disks and the envelopes in the systems, both empirically (Sect. 4.1.1) and through detailed radiative-transer modelling (Sect. 4.1.2). The differences between the two methods and the over-all conclusions are discussed in Sect. 4.1.3.

\subsubsection{Empirical results}

A first-order estimate of the disk and envelope masses can be obtained by assuming that the continuum flux of the envelope is resolved out by the interferometer on the longest baselines (Fig. 2). Hence, the emission on the longest baselines is solely due to the disk, whereas the single-dish flux comes from the disk and envelope combined. Assuming that the disk and the envelope are isothermal and the dust emission is optically thin, the disk mass is given by

$M_{\text {disk }}=\frac{F_{v} \Psi D^{2}}{\kappa_{v} B_{v}\left(T_{\text {dust }}\right)}$,

where $F_{v}$ is the flux at frequency $v$ on the longest baselines, $\Psi$ is the gas-to-dust ratio, $\kappa_{v}$ is the dust opacity at $v$, and $B_{v}\left(T_{\text {dust }}\right)$ is the emission from a black body at $T_{\text {dust }}$. Using fiducial values for all these parameters $-\Psi=100, T_{\text {dust }}=30 \mathrm{~K}$, and $\kappa_{v}=1.18 \mathrm{~cm}^{2} \mathrm{~g}^{-1}$ ("OH5" coagulated dust with icy mantles, found in the fifth column of Table 1 of Ossenkopf \& Henning 1994, interpolated to the observed frequency $v=273 \mathrm{GHz})-$ disk masses of $0.055 M_{\odot}$ and $0.007 M_{\odot}$ are found for IRS 63 and Elias 29, respectively. Using the opacity law as quoted by Beckwith et al. (1990), $\kappa_{v}=10\left(v / 10^{12} \mathrm{~Hz}\right)^{\beta}$, and taking $\beta=1$, a dust opacity at $273 \mathrm{GHz}$ of $\kappa_{v}=2.73 \mathrm{~cm}^{2} \mathrm{~g}^{-1}$ is found, and the disk masses decrease to $0.024 M_{\odot}$ for IRS 63 and $0.003 M_{\odot}$ for Elias $29^{3}$. Note that this method of estimating the disk mass is only valid when the disk is unresolved by the observations.

\footnotetext{
3 The opacity law quoted by Beckwith et al. (1990) is $\kappa_{v}=$ $0.1\left(v / 10^{12} \mathrm{~Hz}\right)^{\beta}$ for the gas and dust combined, implicitly assuming a gas-to-dust ratio $\Psi=100$, consistent with our work.
}

If the disk were clearly resolved, the flux on the longest baselines would not include the emission from the outer disk, but only that from smaller radii, unresolved by those baselines. Due to its closeness, the enhancement in the ridge near Elias 29 may contribute to its flux, even on the longest baselines of $\geq 50 \mathrm{k} \lambda$ or scales $<4$ ". However, in the estimate of the flux on the longest baselines (Table 1), the ridge component was fitted simultaneously with Elias 29, and hence its contribution to the derived disk mass is expected to be minimal.

In a similar fashion the envelope masses in the systems can be found. First, the contributions from the envelopes to the $1.1 \mathrm{~mm}$ single-dish fluxes are determined by subtracting the fluxes at baselines $\geq 16 \mathrm{k} \lambda$ (see Table 1) from the single-dish fluxes as found in Sect. 3. The envelope masses are then found by using Eq. (1), with the temperatures of the envelopes taken to be $20 \mathrm{~K}$. For an optically thin envelope around stars with luminosities of 0.79 and $13.6 L_{\odot}$ applicable to IRS 63 and Elias 29, a temperature of $17-29 \mathrm{~K}$ is expected at a radius of $940 \mathrm{AU}$ corresponding to the size of the JCMT single-dish beam (from, e.g., Eq. (2) of Chandler \& Richer 2000, taking the opacity in$\operatorname{dex} \beta=1$ ). This yields an envelope mass $M_{\mathrm{env}}=0.058 M_{\odot}$ for IRS 63 within a $30^{\prime \prime}$ radius, where the contribution from the envelope to the total $1.1 \mathrm{~mm}$ flux is $38 \%$. Coincidentally, also for Elias 29 an envelope mass $M_{\text {env }}=0.058 M_{\odot}$, within a $20^{\prime \prime}$ radius, is found. However, in this system the contribution from the envelope to the total $1.1 \mathrm{~mm}$ flux is $84 \%$. As for the disk mass, also the envelope mass found for Elias 29 should be taken as an upper limit because of the closeness of the ridge enhancement. Note, however, that it is unlikely that the bulk of the continuum emission is due to this enhancement, because of the coincidence of the continuum peak with the infrared source and also with the single-dish submillimetre positions. Using the opacities of Beckwith et al. (1990) will lower all masses by a factor of about 2 .

\subsubsection{Modelling}

To provide more physical grounding to the values for $M_{\text {disk }}$ and $M_{\text {env }}$ estimated in the previous section, a radiative transfer code was used to fit simultaneously the millimetre interferometry and the spectral energy distributions. IRS 63 and Elias 29 were modelled with a new version of the programme RADMC (e.g., Dullemond \& Dominik 2004). RADMC is an axisymmetric Monte-Carlo code for dust continuum radiative transfer in circumstellar disks and envelopes in which the stellar photons are traced in three dimensions. The code is based on the method of Bjorkman \& Wood (2001). 2008)

The density structure of the disk is given by (see Crapsi et al.

$\rho_{\text {disk }}(r, \theta)=\frac{\Sigma_{0}\left(r / R_{0}\right)^{-1}}{\sqrt{(} 2 \pi) H(r)} \exp \left(-\frac{1}{2}\left[\frac{r \cos (\theta)}{H(r)}\right]^{2}\right)$,

where $r$ is the radial distance from the central star and $\theta$ is the angle from the axis of symmetry. The inner radius was fixed to 0.1 AU for IRS 63, and to 0.25 AU for Elias 29, to account for the higher luminosity of the star. At these radii, the temperature is of the order of the dust sublimation temperature. Note that the exact sublimation temperature, and hence the disk inner radius, depends on the exact dust species and local density. The vertical scale height of the disk is given by $H(r)=r \cdot H_{0} / R_{0} \cdot\left(r / R_{0}\right)^{0.17}$, where $R_{0}$ was left free to vary, and $H_{0} / R_{0}$ was arbitrarily fixed to 0.17 . Chiang \& Goldreich (1997) find $H(r) / r \propto r^{2 / 7}$. However, that estimate is based on grey opacities and constant surface 
Table 2. Model parameters for IRS 63 and Elias 29.

\begin{tabular}{lcc}
\hline \hline & IRS 63 & Elias 29 \\
\hline Modelling parameters and results & & \\
Stellar luminosity (fixed) & $0.79 L_{\odot}$ & $13.6 L_{\odot}$ \\
Disk inner radius (fixed) & $0.1 \mathrm{AU}$ & $0.25 \mathrm{AU}$ \\
Disk outer radius, $R_{0}$ & $100 \mathrm{AU}$ & $200 \mathrm{AU}$ \\
Disk height at outer radius, $H_{0} / R_{0}($ fixed $)$ & 0.17 & 0.17 \\
Envelope outer radius $($ fixed $)$ & $10000 \mathrm{AU}$ & $10000 \mathrm{AU}$ \\
Centrifugal radius, $R_{\text {rot }}$ & $100 \mathrm{AU}$ & $300 \mathrm{AU}$ \\
Inclination & $30^{\circ}$ & $30^{\circ}$ \\
Disk mass, $M_{\text {disk }}$ & $0.13 M_{\odot}$ & $0.004 M_{\odot}$ \\
Envelope mass, $M_{\text {env }}$ & $0.022 M_{\odot}$ & $0.025 M_{\odot}$ \\
\hline Empirical results & & \\
$M_{\text {env }}^{a}\left[M_{\odot}\right]$ & 0.058 & $\leq 0.058$ \\
$M_{\text {disk }}\left[M_{\odot}\right]$ & 0.055 & $\leq 0.007$ \\
$M_{\text {star }}^{b}\left[M_{\odot}\right]$ & $0.37 \pm 0.13$ & $2.5 \pm 0.6$ \\
\hline
\end{tabular}

${ }^{a}$ The envelope mass is defined as the mass, not contained in the disk, within a single-dish radius, i.e., $30^{\prime \prime}$ or $3750 \mathrm{AU}$ for IRS 63 , and $20^{\prime \prime}$ or $2500 \mathrm{AU}$ for Elias 29.

${ }^{b}$ Assuming an inclination $i=30^{\circ}$ (Sect. 4.2 for discussion).

density, and somewhat smaller flaring is usually more realistic. Hence, we chose $H(r) \propto r \cdot r^{0.17} . \Sigma_{0}$ was scaled to the disk mass, which was also left as a free parameter. The envelope density follows the equation for a rotating, infalling model (Ulrich 1976; Crapsi et al. 2008)

$$
\begin{aligned}
\rho_{\text {envelope }}(r, \theta) & =\rho_{0}\left(\frac{r}{R_{\text {rot }}}\right)^{-1.5}\left(1+\frac{\cos \theta}{\cos \theta_{0}}\right)^{0.5} \\
& \times\left(\frac{\cos \theta}{2 \cos \theta_{0}}+\frac{R_{\text {rot }}}{r} \cos ^{2} \theta_{0}\right)^{-1},
\end{aligned}
$$

where $\theta_{0}$ is the solution of the parabolic motion of an infalling particle, $R_{\text {rot }}$ is the centrifugal radius of the envelope, and $\rho_{0}$ is the density in the equatorial plane at $R_{\text {rot }} . \rho_{0}$ was scaled to accomodate the total envelope mass, and $R_{\text {rot }}$, which can have a significant influence on the amplitude as a function of baseline length, was left free to vary. The outer radius of the envelope was fixed to $10000 \mathrm{AU}$, where the temperature is similar to that of the ambient interstellar cloud. The parameters, as summarised in Table 2, give the best-fit models by eye; a full $\chi^{2}$ minimization is not warranted by the relatively low resolution and $S / N$ of the data. Our models do not have outflow cavities in the envelopes. Note that the actual physical structure of the envelope (centrifugal radius, outflow cavities, etc.) may considerably affect the mid-IR SED and thus the derived inclination from the models (e.g., Whitney et al. 2003; Jørgensen et al. 2005).

Two different dust species are used in these models, "hot" dust where the temperature is higher than $90 \mathrm{~K}$, and "cold" dust where the temperature is below $90 \mathrm{~K}$. The opacities used are "OH2" (coagulated dust without ice mantles, Ossenkopf \& Henning 1994) for the hot dust, and "OH5" (coagulated dust with thin ice mantles) for the cold dust. The central stars were represented by sources with luminosities of $0.79 L_{\odot}$ for IRS 63 and 13.6 $L_{\odot}$ for Elias 29, which were taken to be equal to the bolometric luminosities of the sources, and the temperature structures in the disks and envelopes were then calculated by propagating the stellar photons through the systems.

The results of the modelling are shown in Fig. 5. A model with only a $0.055 M_{\odot}$ disk reproduces the amplitude as a function of baseline quite well for IRS 63, but fails to produce enough single-dish flux. A $0.35 M_{\odot}$ envelope-only model, on the other hand, produces enough single-dish flux in the (sub)mm regime,

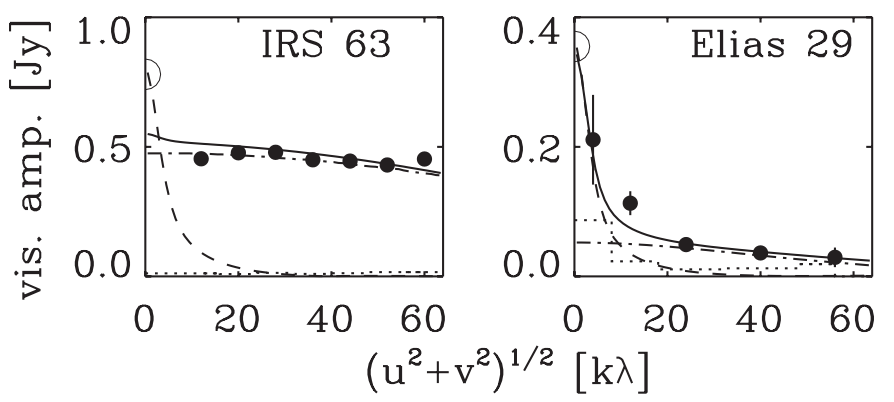

Fig. 5. Continuum amplitude as a function of projected baseline for IRS 63 (left panel) and Elias 29 (right panel), with models overplotted. The dashed lines show models, consisting of an envelope only. They fit the single-dish (sub)mm data well, but fall off too fast to longer projected baselines. The dash-dotted lines are from disk-only models. They fit the interferometric data well, but do not have enough flux on the shortest baselines. The solid lines are for the models that provide a good fit to the SED and to the spatial information provided by the interferometric observations. The dotted lines indicate the expected amplitude for zero signal.

but falls off too quickly toward longer baselines. To allow for a comparison with the empirical model (Sect. 4.1.1) the envelope mass was defined as the mass, present within the singledish aperture (i.e., a 30" radius for IRS 63 and a 20" radius for Elias 29), and not contained in the disk. A model with both a disk and an envelope is necessary to explain the observations of IRS 63, and the best model consists of a disk of $0.13 M_{\odot}$ and an envelope of $0.022 M_{\odot}$. This model also fits the observed IRS 63 SED well, if a foreground extinction of $A_{\mathrm{V}}=7 \mathrm{mag}$ is assumed, see Fig. 6. The region in which IRS 63 is located has a very high $A_{\mathrm{V}}$ of about 24 mag as found in extinction maps produced by the Spitzer "Cores to Disks" Legacy program (Evans et al. 2003, 2007). These maps measure the large scale $\left(\sim 5^{\prime}\right)$ cloud extinction on the basis of background stars, and therefore includes an extra contribution besides envelope extinction.

The amplitude as a function of baseline for Elias 29 is best explained by a $0.004 M_{\odot}$ disk and a $0.025 M_{\odot}$ envelope. For this source, no further attempt to model the SED was made, because the close presence of the ridge enhancement makes a model that is axisymmetric on scales larger than several $100 \mathrm{AU}$ unapplicable.

\subsubsection{Discussion}

Even though the disk and envelope masses derived from the empirical and detailed model results differ by up to a factor of a few, we are confident that they are accurate within this range. For example, a significantly larger disk in the IRS 63 model would show a very steep fall-off in the amplitude as a function of baseline length, which is not observed (Fig. 5). Likewise, an envelope significantly more massive than $0.022 M_{\odot}$ found for IRS 63 would obscure the central star so much that the near- and midIR flux would be severly underestimated (Fig. 6). In the further discussion, we will use the disk and envelope masses as found by the detailed modelling, but note that the overall conclusions do not change if the empirical results are used.

The envelope masses of IRS 63 and Elias 29 are in the same range as those presented by, e.g., Hogerheijde et al. (1997) for a sample of embedded Class I sources in the Taurus-Auriga starforming region. However, the envelope masses of the deeply embedded Class 0 objects are found to be considerably higher, $\gtrsim 1 M_{\odot}$ (Jørgensen et al. 2002; Shirley et al. 2002; Young et al. 2003; Hatchell et al. 2007). While some fraction of sources may 


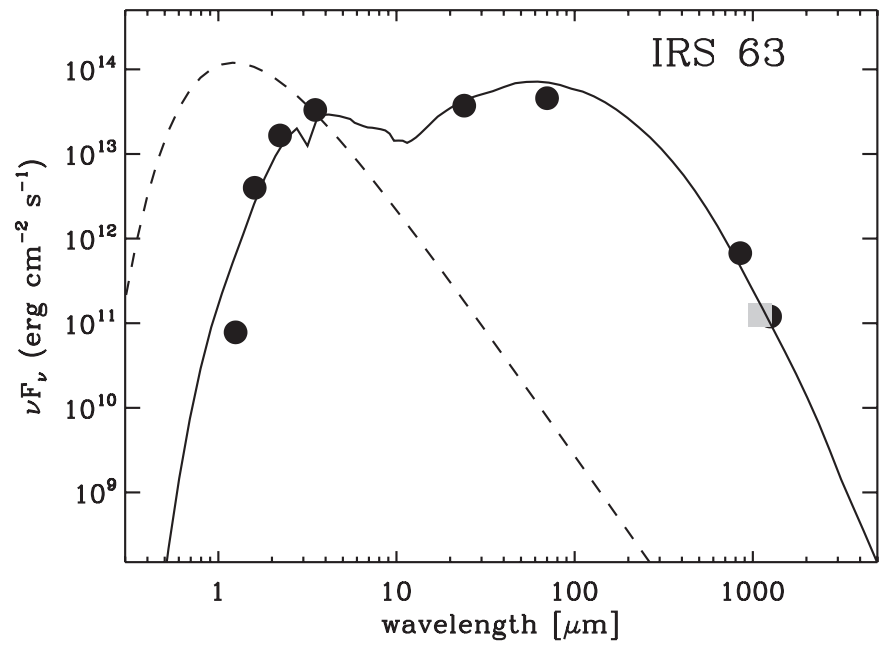

Fig. 6. Spectral energy distribution of IRS 63 using the model parameters of Table 2 extincted by $A_{\mathrm{V}}=7 \mathrm{mag}$ (solid line). The dashed line shows the model star, which has a luminosity of $0.79 L_{\odot}$, and is represented by a black-body with a temperature of $3000 \mathrm{~K}$. Overplotted are JHKL photometry (Haisch et al. 2002), MIPS 24 and $70 \mu \mathrm{m}$ fluxes (c2 $\mathrm{d}^{4}$ ), JCMT $850 \mu \mathrm{m} 60^{\prime \prime}$ integrated flux (COMPLETE), SMA $1.10 \mathrm{~mm}$ peak flux (this work; square), and IRAM $1.25 \mathrm{~mm} 60^{\prime \prime}$ integrated flux (André \& Montmerle 1994).

simply originate from lower mass cores, it is clear that all Class 0 objects must pass through a stage with $M_{\text {env }} \approx 0.1 M_{\odot}$ on their way to the pre-main sequence stage. A larger sample is needed to address the question whether the mass that was initially present in the envelope will first accumulate in the disk, or whether it will pass through the disk and onto the star directly, leaving the disk in a steady state through the entire Class I stage.

An interesting quantity for these YSOs is the ratio $M_{\text {env }} / M_{\text {disk }}$, as this may be a direct measure for their evolutionary stage. The values for the two objects under consideration here0.2 for IRS 63 and 6 for Elias 29 - are quite different, although the value for Elias 29 is rather uncertain due to the contribution of the ridge enhancement to the continuum emission. From these simple arguments, Elias 29 is the less evolved, rather like the deeply embedded Class 0 sources in the PROSAC sample (Jørgensen et al. 2007), which show $M_{\text {env }} / M_{\text {disk }} \gtrsim 10$. IRS 63, on the other hand, is well on its way toward the optically visible Class II or T Tauri stage, which have $M_{\text {env }} \approx 0$. This confirms the notion that the Lada classification is primarily a phenomenological one, not necessarily representing the actual physical stage the YSO is in (see, e.g., Crapsi et al. 2008).

\subsection{Keplerian rotation and stellar masses}

Both IRS 63 and Elias 29 show a velocity gradient in the $\mathrm{HCO}^{+}$ emission, which is interpreted as the rotation of a circumstellar disk. The mass of the central object can be estimated from position-velocity diagrams along the major axis of the disk, as indicated in Fig. 1. An elliptical Gaussian was fitted to each channel in the $\mathrm{HCO}^{+}$data in the $(u, v)$ plane, using the MIRIAD task uvfit. This yielded a best-fit position per channel with corresponding uncertainties in right ascension and declination, and consequently a declination offset from the phase centre in the physical plane. Note that the fitting was carried out in the $(u, v)$ plane, so as not to be hindered by artefacts that may arise in the

\footnotetext{
${ }^{4}$ Delivery of data from the c2d Legacy Project: IRAC and MIPS (Pasadena, SSC, Evans et al. 2007).
}

deconvolution process. Position-velocity diagrams were created by plotting the declination offset as a function of velocity channel, where only coordinates with an uncertainty in the declination of less than 0.'5 were taken into account. Subsequently, the signatures of Keplerian rotation around a point source were fitted to the position-velocity diagrams (Fig. 7). In the fitting process, the values for $M_{\text {star }}$ (the mass of the central object), the central velocity of the system with respect to the local rest frame, and the declination offset from the source to the phase centre were varied, and a global minimum $\chi^{2}$ was determined.

Assuming a Keplerian disk seen edge-on $\left(i=90^{\circ}\right)$, a central mass of $M_{\text {star }}=0.09 \pm 0.03 M_{\odot}$ is found for IRS 63. The exact inclination of IRS 63 is hard to determine due to the high extinction in the region. However, an inclination larger than $\sim 45^{\circ}$ can be ruled out because of the source's brightness at 3-5 $\mu \mathrm{m}$ (Pontoppidan et al. 2003). Assuming an inclination of $30^{\circ}$, and taking into account the $\sin ^{2} i$ dependence, the mass of the central object increases to $M_{\text {star }}=0.37 \pm 0.13 M_{\odot}$. For Elias 29, the points attributed to the emission from the dense ridge (Fig. 7) were disregarded in the fitting procedure. A lower limit to the central mass of $M_{\text {star }}=0.62 \pm 0.14 M_{\odot}$ is found, under the assumption $i=90^{\circ}$. The flatness of the SED limits the inclination of this source to $<60^{\circ}$ (Boogert et al. 2002). A fully face-on orientation, on the other hand, is unlikely, given the presence of low surface brightness scattered K-band light (Zinnecker et al. 1988). An inclination $i=30^{\circ}$ yields a mass for the central source of $2.5 \pm 0.6 M_{\odot}$. With such a high mass, the central star is likely to emerge as a Herbig Ae/Be star, once the surrounding envelope is dissipated.

It is interesting to compare the inferred masses to those inferred for optically visible pre-main sequence stars. With a stellar mass of $0.37 M_{\odot}$ and a stellar luminosity of $0.79 L_{\odot}$, IRS 63 would be found to have an age of about $5 \times 10^{5} \mathrm{yr}$, when compared to evolutionary tracks of classical and weak-lined T Tauri stars in the $\rho$ Ophiuchi cloud (Wilking et al. 2005; D'Antona \& Mazzitelli 1997). A similar age would be found for Elias 29 (e.g., Palla \& Stahler 1993).

\section{Conclusions}

We used the SMA to observe the Class I YSOs Elias 29 and IRS 63 at $1.1 \mathrm{~mm}$. The main results are as follows.

- Both sources are detected in the continuum and in the $\mathrm{HCO}^{+}$ $J=3-2$ line, but not in the $\mathrm{HCN} J=3-2$ line. The $\mathrm{HCO}^{+}$ emission toward IRS 63 is compact and associated with the YSO, whereas a significant part of the molecular emission in the direction of Elias 29 is due to an enhancement in the ridge from which Elias 29 formed.

- Assuming that the continuum emission toward the sources is optically thin, and that any circumstellar envelope is resolved out by the interferometer on the longest baselines, we find empirical disk masses of 0.055 for IRS 63 and $\leq 0.007 M_{\odot}$ for Elias 29. By comparing our interferometer data with singledish observations, envelope masses of $0.058 M_{\odot}$ are derived for both sources, within $30^{\prime \prime}$ radius for IRS 63 and 20" radius for Elias 29, where the value for Elias 29 should be treated as an upper limit because of the presence of the ridge enhancement.

- Using a radiative-transfer code to create SEDs and amplitude vs. $(u, v)$-distance plots for different combinations of disk and envelope masses, we find disk masses of $0.13 M_{\odot}$ for IRS 63 and $0.004 M_{\odot}$ for Elias 29, and envelope masses of $0.022 M_{\odot}$ and $0.025 M_{\odot}$. 

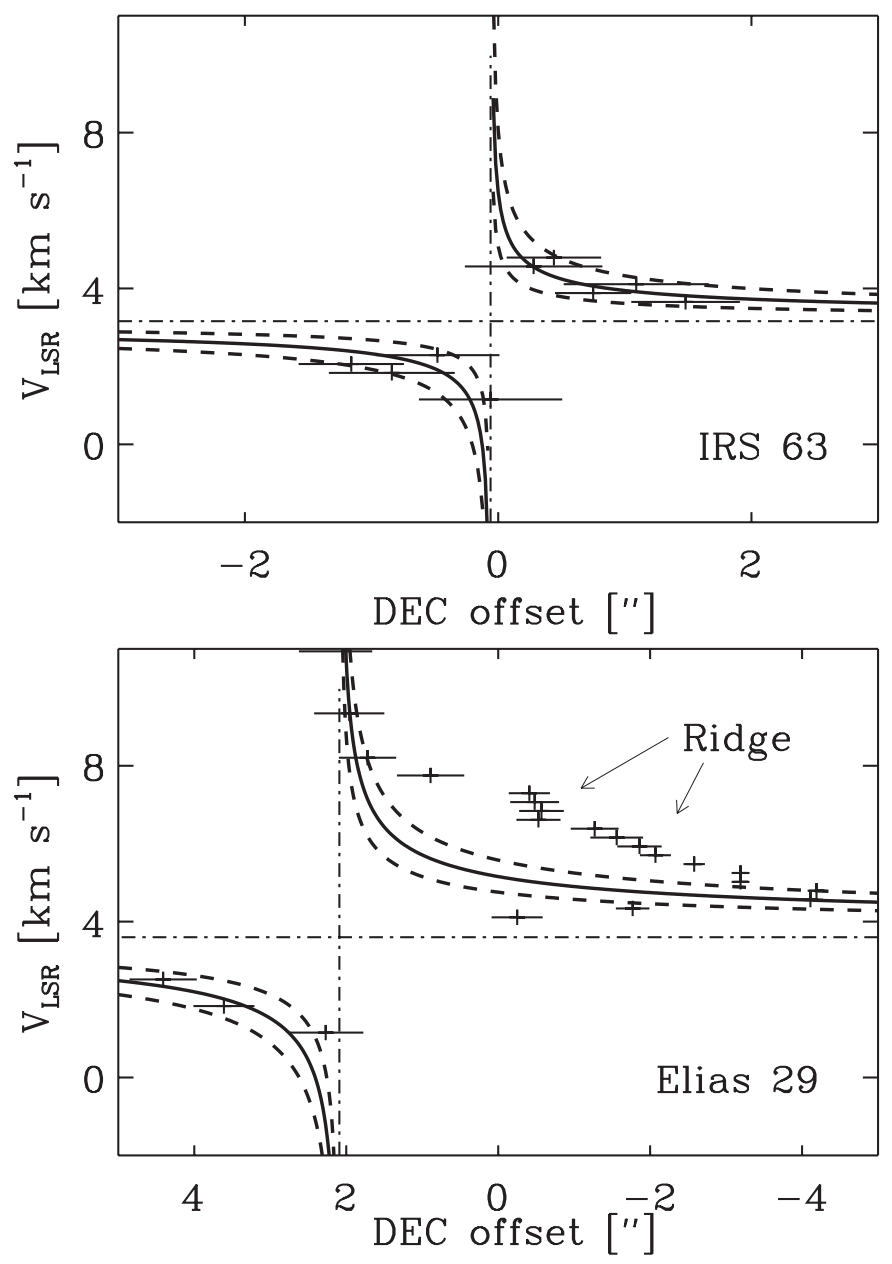

Fig. 7. Velocity of the $\mathrm{HCO}^{+} J=3-2$ line as a function of the declination offset from the phase centre for IRS 63 (upper panel) and Elias 29 (lower panel). The solid lines show the results from $\chi^{2}$ fits to the data, yielding central masses of $0.37 M_{\odot}$ (IRS 63) and $2.5 M_{\odot}$ (Elias 29) for inclinations of $30^{\circ}$; the dashed lines show formal $99 \%$ confidence intervals. Points attributed to the emission from the dense ridge are indicated and are not included in the fit. The declination offsets are different for the two panels.

- The modelling results yield rather different ratios of $M_{\text {env }} / M_{\text {disk }}: 0.2$ for IRS 63 and 6 for Elias 29, with uncertainties of a factor of a few. From this it is concluded that IRS 63 is well on its way to become an optically visible Class II source, rather than an embedded Class I source. Elias 29, on the other hand, is more like the deeply embedded Class 0 sources.

- Velocity gradients in the $\mathrm{HCO}^{+} J=3-2$ emission are interpreted as signs of Keplerian rotation, and indicate central masses of $2.5 \pm 0.6 M_{\odot}$ for Elias 29 and $0.37 \pm 0.13 M_{\odot}$ for IRS 63 , for inclinations of $30^{\circ}$. These masses correspond to ages of a few $10^{5} \mathrm{yr}$.

With a larger set of sources treated in a similar manner as is presented for IRS 63 and Elias 29 here, it will be possible to constrain models for the evolution of the envelope, disk, and stellar masses through this important stage of YSOs.
Acknowledgements. We would like to thank the anonymous referee for a very constructive report, which greatly improved the paper. Demerese Salter and Doug Johnstone are thanked for providing us with data from JCMT SCUBA observations. The discussions with the members of the Leiden "AstroChem" group were very useful. We are grateful to Kees Dullemond for providing us with the most recent version of the RADMC package. Partial support for this work was provided by a Netherlands Research School For Astronomy network 2 grant, and by a Netherlands Organisation for Scientific Research Spinoza grant.

\section{References}

André, P., \& Montmerle, T. 1994, ApJ, 420, 837

André, P., Ward-Thompson, D., \& Barsony, M. 1993, ApJ, 406, 122

Andrews, S. M., \& Williams, J. P. 2007, ApJ, 659, 705

Beckwith, S. V. W., Sargent, A. I., Chini, R. S., \& Guesten, R. 1990, AJ, 99, 924

Benson, P. J., \& Myers, P. C. 1989, ApJS, 71, 89

Bjorkman, J. E., \& Wood, K. 2001, ApJ, 554, 615

Boogert, A. C. A., Hogerheijde, M. R., Ceccarelli, C., et al. 2002, ApJ, 570, 708

Chandler, C. J., \& Richer, J. S. 2000, ApJ, 530, 851

Chiang, E. I., \& Goldreich, P. 1997, ApJ, 490, 368

Crapsi, A., van Dishoeck, E. F., Hogerheijde, M. R., Pontoppidan, K. M., \& Dullemond, C. P. 2008, A\&A, in press.

D’Antona, F., \& Mazzitelli, I. 1997, Mem. Soc. Astron. Ital., 68, 807

de Geus, E. J., de Zeeuw, P. T., \& Lub, J. 1989, A\&A, 216, 44

Dullemond, C. P., \& Dominik, C. 2004, A\&A, 417, 159

Evans, N. J., Allen, L. E., Blake, G. A., et al. 2003, PASP, 115, 965

Evans, II, N. J., Harvey, P. M., Dunham, M. M., et al. 2007, Delivery of Data from the c2d Legacy Project: IRAC and MIPS, Pasadena, SSC

Flaherty, K. M., Pipher, J. L., Megeath, S. T., et al. 2007, ApJ, 663, 1069

Greene, T. P., Wilking, B. A., Andre, P., Young, E. T., \& Lada, C. J. 1994, ApJ, 434, 614

Haisch, Jr., K. E., Barsony, M., Greene, T. P., \& Ressler, M. E. 2002, AJ, 124, 2841

Harvey, D. W. A., Wilner, D. J., Myers, P. C., \& Tafalla, M. 2003, ApJ, 596, 383 Hatchell, J., Fuller, G. A., Richer, J. S., Harries, T. J., \& Ladd, E. F. 2007, A\&A, 468, 1009

Ho, P. T. P., Moran, J. M., \& Lo, K. Y. 2004, ApJ, 616, L1

Hogerheijde, M. R., van Dishoeck, E. F., Blake, G. A., \& van Langevelde, H. J. 1997, ApJ, 489, 293

Hogerheijde, M. R., van Dishoeck, E. F., Blake, G. A., \& van Langevelde, H. J. 1998, ApJ, 502, 315

Johnstone, D., Di Francesco, J., \& Kirk, H. 2004, ApJ, 611, L45

Jørgensen, J. K., Schöier, F. L., \& van Dishoeck, E. F. 2002, A\&A, 389, 908

Jørgensen, J. K., Hogerheijde, M. R., van Dishoeck, E. F., Blake, G. A., \& Schöier, F. L. 2004, A\&A, 413, 993

Jørgensen, J. K., Bourke, T. L., Myers, P. C., et al. 2005, ApJ, 632, 973

Jørgensen, J. K., Bourke, T. L., Myers, P. C., et al. 2007, ApJ, 659, 479

Lada, C. J. 1987, in Star Forming Regions, ed. M. Peimbert, \& J. Jugaku, IAU Symp., 115, 1

Lada, C. J., \& Wilking, B. A. 1984, ApJ, 287, 610

Looney, L. W., Mundy, L. G., \& Welch, W. J. 2000, ApJ, 529, 477

Ohashi, N., Hayashi, M., Ho, P. T. P., \& Momose, M. 1997, ApJ, 475, 211

Ossenkopf, V., \& Henning, T. 1994, A\&A, 291, 943

Palla, F., \& Stahler, S. W. 1993, ApJ, 418, 414

Pontoppidan, K. M., Fraser, H. J., Dartois, E., et al. 2003, A\&A, 408, 981

Qi, C. 2005, The MIR Cookbook, The Submillimeter Array / HarvardSmithsonian Center for Astrophysics http://cfa-www.harvard.edu/ $\sim$ cqi/mircook. html

Ridge, N. A., Di Francesco, J., Kirk, H., et al. 2006, AJ, 131, 2921

Sault, R. J., Teuben, P. J., \& Wright, M. C. H. 1995, in Astronomical Data Analysis Software and Systems IV, ASP Conf. Ser., 77, 433

Shirley, Y. L., Evans, II, N. J., \& Rawlings, J. M. C. 2002, ApJ, 575, 337

Ulrich, R. K. 1976, ApJ, 210, 377

Whitney, B. A., Wood, K., Bjorkman, J. E., \& Wolff, M. J. 2003, ApJ, 591, 1049 Wilking, B. A., Meyer, M. R., Robinson, J. G., \& Greene, T. P. 2005, AJ, 130, 1733

Young, C. H., Shirley, Y. L., Evans, II, N. J., \& Rawlings, J. M. C. 2003, ApJS, 145,111

Zinnecker, H., Perrier, C., \& Chelli, A. 1988, in Proc. NOAO-ESO Conference on High-Resolution Imaging by Interferometry: Ground-Based Interferometry at Visible and Infrared Wavelengths, ed. F. Merkle 